\title{
Isotone $\mathcal{L}$-Fuzzy Formal Concept Analysis and $\mathcal{L}$-Valued Fuzzy Measures and Integrals
}

\author{
Ondrej Krídlo ${ }^{(\varpi)}(\mathbb{0}$ \\ P.J. Šafárik University in Košice, Košice, Slovakia \\ ondrej.kridlo@upjs.sk
}

\begin{abstract}
The main idea of the paper is to generalize the concept of lattice valued fuzzy measures and integrals for data from complete residuated lattice where double negation law holds and then to show their relationship to isotone $\mathcal{L}$-fuzzy concept forming operators.
\end{abstract}

Keywords: Fuzzy measure $\cdot$ Fuzzy integral $\cdot$ Formal concept analysis

\section{Introduction}

Once I saw a very nice presentation about fuzzy sets and its applications and basic technics where very simple but nice toy example of fuzzy measure and fuzzy integral were presented. It was my almost first meeting with such an area where I found some small intuitive connection with fuzzy Formal concept analysis (FCA) $[2,3,5,6,12,13]$ that is my main topic for years. After that experience my scientific curiosity led me to papers that seemed to me as a newcomer as most understandable $[1,7,14-17]$ and also very useful was to read two papers that deals with both fuzzy integrals and fuzzy FCA $[4,8]$.

Main topic of the paper is to first connect two notions, lattice valued fuzzy measures and integrals [17] and t-norm and t-conorm fuzzy measures and integrals [16] into one "complete residuated lattice"-valued fuzzy measures and integrals, where double negation law has to be preserved. Next step is to show their connection with isotone derivation (concept forming) operators of fuzzy formal context that are the most important part of FCA.

Second section is dedicated to basics of isotone FCA based on data from complete residuated lattice. Third section is about proposition of so called one-sided fuzzy concept-forming operators and the definition of new complete residuated lattice valued fuzzy measures and integrals. All needed and some new properties are proved.

This article was created in the framework of the National project IT Academy - Education for the 21st Century, which is supported by the European Social Fund and the European Regional Development Fund in the framework of the Operational Programme Human Resources. Supported also by the Slovak Research and Development Agency contract No. APVV-15-0091.

(C) Springer Nature Switzerland AG 2020

M.-J. Lesot et al. (Eds.): IPMU 2020, CCIS 1239, pp. 726-735, 2020.

https://doi.org/10.1007/978-3-030-50153-2_53 


\section{Isotone $\mathcal{L}$-fuzzy Formal Concept Analysis}

Definition 1. An algebra $\mathcal{L}=\langle L, \wedge, \vee, 0,1, \otimes, \rightarrow\rangle$ is said to be a complete residuated lattice if

1. $\langle L, \wedge, \vee, 0,1\rangle$ is a complete lattice where 0 and 1 are the bottom and top elements (resp.).

2. $\langle L, \otimes, 1\rangle$ is a commutative monoid.

3. $\langle\otimes, \rightarrow\rangle$ is an adjoint pair, i.e. $k \otimes m \leq n$ if and only if $k \leq m \rightarrow n$, for all $k, m, n \in L$, where $\leq$ is the ordering generated by $\wedge$ and $\vee$.

It will be important to have $\mathcal{L}$ with double neggation law, ie. $\neg \neg k=k$ for any $k \in L$, where $\neg k=k \rightarrow 0$.

Definition 2. A Girard monoid is a residuated lattice $\mathcal{L}$ satisfying the law of double negation, namely, the equality $x=(x \rightarrow 0) \rightarrow 0$ holds for all $x \in L$.

This notion represents one of the several flavours in which one can find residuated lattices, it was used by Girard in his development programme for linear logics, and a study of its structure in the particular case of the unit interval can be found in [9]. Other well-known enriched versions of residuated lattices include, for instance, Heyting algebras (satisfying $x \otimes y=x \wedge y$ ), BL-algebras (satisfying divisibility, i.e. $x \wedge y=x \otimes(x \rightarrow y)$, and the prelinearity, i.e. $(x \rightarrow y) \vee(y \rightarrow$ $x)=1$ ), or MV-algebras (BL-algebra satisfying the law of double negation).

Definition 3. The operator $\oplus: L \times L \rightarrow L$ is defined by

$$
a \oplus b=\neg a \rightarrow b=(a \rightarrow 0) \rightarrow b .
$$

Assuming that we are working on a Girard monoid, it is not difficult to check that $\oplus$ is commutative and associative. Furthermore, the De Morgan laws between $\otimes$ and $\oplus$, and also between $\vee$ and $\wedge$, and contraposition law also hold. Hereafter, we will assume that $\mathcal{L}$ is a Girard monoid.

Let $X$ be any final set. The ordered set (complete lattice) of all $\mathcal{L}$-sets over $X$ will be denoted by $\mathcal{L}^{X}$.

Definition 4. $\mathcal{L}$-Fuzzy Formal Context is a triple $\langle B, A, \mathcal{L}, r\rangle$ where $B$ is the set of objects, $A$ is the set of attributes and $r: B \times A \rightarrow \mathcal{L}$ is a binary relation between objects and attributes.

Definition 5. Let us define two pairs of so called isotone derivation operators between ( $\mathcal{L}$-fuzzy) powerset complete lattices over the sets of objects and attributes as follows:

1. $\quad-\nearrow: \mathcal{L}^{B} \longleftarrow \mathcal{L}^{A}$ and $\swarrow: \mathcal{L}^{A} \longleftarrow \mathcal{L}^{B}$.

- $(f)(a)=\bigvee_{b \in B}(f(b) \otimes r(b, a))$ for any $f \in \mathcal{L}^{B}$.

$-\swarrow(g)(b)=\bigwedge_{a \in A}(r(b, a) \rightarrow g(a))$ for any $g \in \mathcal{L}^{A}$.

2. $-\nwarrow: \mathcal{L}^{B} \longleftarrow \mathcal{L}^{A}$ and $\searrow: \mathcal{L}^{A} \longleftarrow \mathcal{L}^{B}$.

$-\nwarrow(f)(a)=\bigwedge_{b \in B}(r(b, a) \rightarrow f(b))$ for any $f \in \mathcal{L}^{B}$. 


$$
\text { - }(g)(b)=\bigvee_{a \in A}(g(a) \otimes r(b, a)) \text { for any } g \in \mathcal{L}^{A} .
$$

For above defined operators holds $(\swarrow, \nearrow)$ and $(\searrow, \nwarrow)$ form an isotone Galois connections between $\mathcal{L}^{B}$ and $\mathcal{L}^{A}$, i.e. for any $f \in \mathcal{L}^{B}$ and $g \in \mathcal{L}^{A}$ holds

$$
\nearrow(f) \leq g \quad \Leftrightarrow \quad f \leq \swarrow(g) \quad \text { and } \quad g \leq \nwarrow(f) \quad \Leftrightarrow \quad \searrow(g) \leq f .
$$

As a consequence of previous facts the compositions

- $\swarrow \nearrow$ and $\nwarrow \searrow$ forms closure operators on $\mathcal{L}^{B}$ or $\mathcal{L}^{A}$ respectively.

- $\nearrow$ and $\searrow \nwarrow$ forms interior operator on $\mathcal{L}^{A}$ or $\mathcal{L}^{B}$ respectively.

That means that

- all compositions are monotone

$-\swarrow \nearrow$ and $\nwarrow \searrow$ are inflationary (i.e. $\nwarrow \searrow(g) \geq g$ for any $g \in \mathcal{L}^{A}$ )

$-\nearrow \swarrow$ and $\searrow \nwarrow$ are deflationary (i.e. $\searrow \nwarrow(f) \leq f$ for any $f \in \mathcal{L}^{B}$ ).

- all compositions are idempotent (i.e. $\swarrow \nearrow \swarrow \nearrow(f)=\swarrow \nearrow(f)$ for any $f \in \mathcal{L}^{B}$ )

Pairs $(f, g) \in \mathcal{L}^{B} \times \mathcal{L}^{A}$ such that $\nearrow(f)=g$ and $\swarrow(g)=f$ is called $\mathcal{L}$ fuzzy formal concept of $\mathcal{L}$-context $\langle B, A, \mathcal{L}, r\rangle$ with respect to operators $(\nearrow, \swarrow)$. $f$ is then called extent and $g$ is called intent. All extents will be denoted by $\operatorname{Ext}(B, A, \mathcal{L}, r, \nearrow, \swarrow)$ and all intents by $\operatorname{Int}(B, A, \mathcal{L}, r, \nearrow, \swarrow)$. Similarly for the other pair of operators $(\nwarrow, \searrow)$. Sets of all extents and intents ordered by $\mathcal{L}$-fuzzy set inclusion form complete lattices.

\section{$3 \quad \mathcal{L}$-fuzzy Isotone Derivation Operators and $\mathcal{L}$-fuzzy Measures and Integrals}

Krajči in $[10,11]$ defined a modification of antitone $\mathcal{L}$-fuzzy derivation operators $(\uparrow, \downarrow)$, mappings between $\mathcal{L}^{B}$ and $\mathcal{L}^{A}$ into the case of mappings between $2^{B}$ and $\mathcal{L}^{A}$, due to better understanding or interpretation of possible results.

So now the isotone derivation operators will be modified into one sided form:

Let $X \subseteq B$ be an arbitrary classical subset. $\chi_{X}$ is a characteristic function of $X$. Let us define a new mappings $\bar{\nearrow}: 2^{B} \rightarrow \mathcal{L}^{A}$ and $\bar{\nwarrow}: 2^{B} \rightarrow \mathcal{L}^{A}$

$$
\begin{aligned}
\bar{\nearrow}(X)(a) & =\nearrow\left(\chi_{X}\right)(a)=\bigvee_{b \in B} \chi_{X}(b) \otimes r(b, a) \\
& =\bigvee_{b \in X} 1 \otimes r(b, a) \vee \bigvee_{b \in X^{\mathrm{c}}} 0 \otimes r(b, a) \\
& =\bigvee_{b \in X} 1 \otimes r(b, a) \vee 0=\bigvee_{b \in X} r(b, a)
\end{aligned}
$$




$$
\begin{aligned}
\bar{\nwarrow}(X)(a) & =\nwarrow\left(\chi_{X}\right)(a)=\bigwedge_{b \in B}\left(r(b, a) \rightarrow \chi_{X}(b)\right) \\
& =\bigwedge_{b \in X}(r(b, a) \rightarrow 1) \wedge \bigwedge_{b \in X^{\mathrm{c}}}(r(b, a) \rightarrow 0) \\
& =1 \wedge \bigwedge_{b \in X^{\mathrm{c}}}(r(b, a) \rightarrow 0)=\bigwedge_{b \in X^{\mathrm{c}}} \neg r(b, a)
\end{aligned}
$$

Definition 6. Let $B$ be an arbitrary set and $\mathcal{L}$ be the lattice with bottom 0 and top element 1 . The mapping $\mu: \mathcal{P}(B) \rightarrow \mathcal{L}$ is called $\mathcal{L}$-valued fuzzy measure iff $\mu$ is monotone, $\mu(\emptyset)=0$ and $\mu(B)=1$.

Before the theorem about a new measure, let us define a notion of normal $\mathcal{L}$-set and normal $\mathcal{L}$-context.

Definition 7. Any $\mathcal{L}$-set from $\mathcal{L}^{X}$ is called normal when its maximal membership value is equal to 1 (top of $\mathcal{L}$ ). Let $\langle B, A, \mathcal{L}, r\rangle$ be a $\mathcal{L}$-fuzzy formal context, such that its columns $r(-, a)$ are normal $\mathcal{L}$-fuzzy sets from $\mathcal{L}^{B}$. Such $\mathcal{L}$-context is also called normal.

Theorem 1. Let $\langle B, A, \mathcal{L}, r\rangle$ be a normal $\mathcal{L}$-fuzzy formal context. Let $a \in A$ be arbitrary attribute. The following upper and lower $\mathcal{L}$-valued mappings

- $\underline{\mu}_{a}$ where for any $X \subseteq B$ is $\underline{\mu}_{a}(X)=\bar{\nearrow}(X)(a)$

- $\bar{\mu}_{a}$ where for any $X \subseteq B$ is $\bar{\mu}_{a}(X)=\bar{\nwarrow}(X)(a)$.

are $\mathcal{L}$-valued fuzzy measures.

Proof. It is well known that operators $\nearrow$ and $\nwarrow$ are monotone, hence also their one-sided fuzzy form will also be monotone. Moreover

$$
\bar{\mu}_{a}(B)=\bigwedge_{b \in B^{\mathrm{c}}} \neg r(b, a)=1 \quad \text { and } \quad \underline{\mu}_{a}(\emptyset)=\bigvee_{b \in \emptyset} r(b, a)=0
$$

for any $a \in A$.

What can be little questionable are the following two facts. In general $\bar{\mu}_{a}(\emptyset)$ need not to be equal to 0 and $\underline{\mu}_{a}(B)$ need not to be equal to 1 . This is why the precondition of normality is here, which says that there for any $a \in A$ there exists at least one $b \in B$ such that $r(b, a)=1$. Hence

$$
\bar{\mu}_{a}(\emptyset)=\bigwedge_{b \in \emptyset c} \neg r(b, a)=\bigwedge_{b \in B} \neg r(b, a)=0 \quad \text { and } \quad \underline{\mu}_{a}(B)=\bigvee_{b \in B} r(b, a)=1
$$

Before the definition of integral let us first define some auxiliary notation. Let $f \in \mathcal{L}^{B}$ be an arbitrary $\mathcal{L}$-set over $B$. Then 
- $f_{\alpha}=\{b \in B \mid f(b) \geq \alpha\}$ is well known $\alpha$-cut of $\mathcal{L}$-set for some $\alpha \in \mathcal{L}$

- $f^{\alpha}=\{b \in B \mid f(b) \not \leq \alpha\}$

The following definition is a generalisation of the definition of lower and upper-lattice valued fuzzy integrals from [17].

Theorem 2. Let $\langle B, A, \mathcal{L}, r\rangle$ be the normal $\mathcal{L}$-fuzzy formal context and for each $a \in A$ there are corresponding $\underline{\mu}_{a}$ and $\bar{\mu}_{a}$ lower and upper $\mathcal{L}$-fuzzy measures. The following lower and upper $\overline{\mathcal{L}}$-valued mappings defined for any $f \in \mathcal{L}^{B}$ and $X \subseteq B$ as follows

$$
\underline{\int}_{X} f d \underline{\mu}_{a}=\bigvee_{\alpha \in \mathcal{L}} \alpha \otimes \underline{\mu}_{a}(f \alpha \cap X) \quad \text { and } \quad \bar{\int}_{X} f d \bar{\mu}_{a}=\bigwedge_{\alpha \in \mathcal{L}} \alpha \oplus \bar{\mu}_{a}\left(f^{\alpha} \cap X\right)
$$

are lower and upper $\mathcal{L}$-valued fuzzy integrals.

Proof. In [14] where the definition of fuzzy integral says that it has to be idempotent homeomorphism. So we have to prove for above defined $\mathcal{L}$-valued mappings the following properties:

1. they are monotone

2. they map top $\mathcal{L}$-set from $\mathcal{L}^{B}$ to 1 and bottom to 0

3. for any $\gamma \in \mathcal{L}$ a constant $\mathcal{L}$-set $\bar{\gamma}$ defined as $\bar{\gamma}(b)=\gamma$ for any $b \in B$ holds:

$$
\begin{aligned}
& -\int_{B} \bar{\gamma} d \underline{\mu}_{a}=\gamma \\
& -\bar{\int}_{B} \bar{\gamma} d \bar{\mu}_{a}=\gamma
\end{aligned}
$$

1. The monotonicity follows directly from the definition of integrals.

2. From interior and closure operator properties of $\nearrow$ and $\swarrow$ from the Sect. 2 and the normality precondition the following holds.

$$
\begin{aligned}
& \underline{\int}_{B} \chi_{B} d \underline{\mu}_{a}=\bigvee_{\alpha \in \mathcal{L}} \alpha \otimes \underline{\mu}_{a}(B)=\bigvee_{\alpha \in \mathcal{L}} \alpha \otimes 1=1 \otimes 1=1 \\
& \underline{\int}_{B} \chi_{\emptyset} d \underline{\mu}_{a}=\bigvee_{\alpha \in \mathcal{L}} \alpha \otimes \underline{\mu}_{a}(\emptyset)=\bigvee_{\alpha \in \mathcal{L}} \alpha \otimes 0=0 \\
& \int_{B} \chi_{B} d \bar{\mu}_{a}(B)=\bigwedge_{\alpha \in L L} \alpha \oplus \bar{\mu}_{a}(B)=\bigwedge_{\alpha \in \mathcal{L}} \alpha \oplus 1=1 \\
& \bar{\int}_{B} \chi_{B} d \bar{\mu}_{a}(\emptyset)=\bigwedge_{\alpha \in L L} \alpha \oplus \bar{\mu}_{a}(\emptyset)=\bigwedge_{\alpha \in \mathcal{L}} \alpha \oplus 0=0 \oplus 0=0
\end{aligned}
$$


3.

$$
\begin{aligned}
& \underline{\int}_{B} \bar{\gamma} d \underline{\mu}_{a}=\bigvee_{\alpha \in \mathcal{L}} \alpha \otimes \underline{\mu}_{a}\left(B \cap \bar{\gamma}_{\alpha}\right) \\
& =\bigvee_{\alpha \in \mathcal{L} ; \alpha \leq \gamma} \alpha \otimes \underline{\mu}_{a}\left(B \cap \bar{\gamma}_{\alpha}\right) \vee \bigvee_{\alpha \in \mathcal{L} ; \alpha \underline{\not} \gamma} \alpha \otimes \underline{\mu}_{a}\left(B \cap \bar{\gamma}_{\alpha}\right) \\
& =\bigvee_{\alpha \in \mathcal{L} ; \alpha \leq \gamma} \alpha \otimes \underline{\mu}_{a}(B) \vee \bigvee_{\alpha \in \mathcal{L} ; \alpha \not \gamma \gamma} \alpha \otimes \underline{\mu}_{a}(\emptyset) \\
& =\gamma \otimes \underline{\mu}_{a}(B) \vee \bigvee_{\alpha \in \mathcal{L} ; \alpha \mathbb{\leqslant} \gamma} \alpha \otimes 0 \\
& =(\gamma \otimes 1) \vee 0=\gamma \\
& \int_{B} \bar{\gamma} d \bar{\mu}_{a}=\bigwedge_{\alpha \in \mathcal{L}} \alpha \oplus \bar{\mu}_{a}\left(B \cap \bar{\gamma}^{\alpha}\right) \\
& =\bigwedge_{\alpha \in \mathcal{L} ; \alpha \leq \gamma} \alpha \oplus \bar{\mu}_{a}\left(B \cap \bar{\gamma}^{\alpha}\right) \wedge \bigwedge_{\alpha \in \mathcal{L} ; \alpha \mathbb{z} \gamma} \alpha \oplus \bar{\mu}_{a}\left(B \cap \bar{\gamma}^{\alpha}\right) \\
& =\bigwedge_{\alpha \in \mathcal{L} ; \alpha \leq \gamma} \alpha \oplus \bar{\mu}_{a}(\emptyset) \wedge \bigwedge_{\alpha \in \mathcal{L} ; \alpha \not \gamma \gamma} \alpha \oplus \bar{\mu}_{a}(B) \\
& =\gamma \oplus \bar{\mu}_{a}(\emptyset) \wedge \bigwedge_{\alpha \in \mathcal{L} ; \alpha \nsubseteq \gamma} \alpha \oplus 1 \\
& =\gamma \wedge 1=\gamma
\end{aligned}
$$

The following theorem will show a relationship between new integrals and concept forming operators.

Theorem 3. Let $\langle B, A, \mathcal{L}, r\rangle$ be a $\mathcal{L}$-context and $\left\{\underline{\mu}_{a} \mid a \in A\right\}$ and $\left\{\bar{\mu}_{a} \mid a \in A\right\}$ be its collections of measures. Then for corresponding integrals holds:

$$
\underline{\int}_{B} f d \underline{\mu}_{a}=\nearrow(f)(a) \text { and } \quad \int_{B} f d \bar{\mu}_{a}=\nwarrow(f)(a) .
$$

Proof. Let $f$ be an arbitrary from $\mathcal{L}^{B}$.

$$
\begin{aligned}
\underline{\int}_{B} f d \underline{\mu}_{a} & =\bigvee_{\alpha \in \mathcal{L}} \alpha \otimes \underline{\mu}_{a}\left(B \cap f_{\alpha}\right) \\
& =\bigvee_{\alpha \in \mathcal{L}} \alpha \otimes \bigvee_{b \in f_{\alpha}} r(b, a) \\
& =\bigvee_{\alpha \in \mathcal{L}} \alpha \otimes \bigvee_{b \in B ; f(b) \geq \alpha} r(b, a)
\end{aligned}
$$




$$
\begin{aligned}
= & \bigvee_{\alpha \in \mathcal{L}} \bigvee_{b \in B ; f(b) \geq \alpha} \alpha \otimes r(b, a) \\
= & \bigvee_{b \in B} \bigvee_{\alpha \in \mathcal{L} ; f(b) \geq \alpha} \alpha \otimes r(b, a) \\
= & \bigvee_{b \in B} f(b) \otimes r(b, a)=\nearrow(f)(a) \\
\int_{B} f d \bar{\mu}_{a} & =\bigwedge_{\alpha \in \mathcal{L}} \alpha \oplus \bar{\mu}_{a}\left(B \cap f^{\alpha}\right) \\
& =\bigwedge_{\alpha \in \mathcal{L}} \neg \alpha \rightarrow \bigwedge_{b \in\left(f^{\alpha}\right)^{c}} \neg r(b, a) \\
& =\bigwedge_{\alpha \in \mathcal{L}} \bigwedge_{b \in B ; f(b) \leq \alpha} \neg \alpha \rightarrow \neg r(b, a) \\
& =\bigwedge_{\alpha \in \mathcal{L}} \bigwedge_{b \in B ; f(b) \leq \alpha} r(b, a) \rightarrow \alpha \\
& =\bigwedge_{b \in B} \bigwedge_{\alpha \in \mathcal{L} ; f(b) \leq \alpha} r(b, a) \rightarrow \alpha \\
& =\bigwedge_{b \in B} r(b, a) \rightarrow f(b)=\nwarrow(f)(a)
\end{aligned}
$$

Theorem 4. Let $\langle B, A, \mathcal{L}, r\rangle$ be a $\mathcal{L}$-context and $\left\{\underline{\mu}_{a} \mid a \in A\right\}$ and $\left\{\bar{\mu}_{a} \mid a \in A\right\}$ be its collections of measures. Then for corresponding integrals holds:

$$
\underline{\int}_{X} f d \underline{\mu}_{a}=\nearrow\left(f \cap \chi_{X}\right)(a) \text { and } \bar{\int}_{X} f d \bar{\mu}_{a}=\nwarrow\left(f \cap \chi_{X}\right)(a)
$$

Proof. Let $f$ be an arbitrary from $\mathcal{L}^{B}$ and $X \subseteq B$.

$$
\begin{aligned}
\underline{f}_{X} f d \underline{\mu}_{a} & =\bigvee_{\alpha \in \mathcal{L}} \alpha \otimes \underline{\mu}_{a}\left(f_{\alpha} \cap X\right) \\
& =\bigvee_{\alpha \in \mathcal{L}} \alpha \otimes \bigvee_{b \in f_{\alpha} \cap X} r(b, a) \\
& =\bigvee_{\alpha \in \mathcal{L}} \bigvee_{b \in f_{\alpha} \cap X} \alpha \otimes r(b, a) \\
& =\bigvee_{\alpha \in \mathcal{L}} \bigvee_{b \in X, f(b) \geq \alpha} \alpha \otimes r(b, a) \\
& =\bigvee_{b \in X} \bigvee_{\alpha \in \mathcal{L}, \alpha \leq f(b)} \alpha \otimes r(b, a)
\end{aligned}
$$




$$
\begin{aligned}
& =\bigvee_{b \in X} f(b) \otimes r(b, a) \\
& =\bigvee_{b \in B}\left(f(b) \wedge \chi_{X}(b)\right) \otimes r(b, a) \\
& =\nearrow\left(f \cap \chi_{X}\right)(a)
\end{aligned}
$$

$$
\begin{aligned}
& \bar{\int}_{X} f d \bar{\mu}_{a}=\bigwedge_{\alpha \in \mathcal{L}} \alpha \oplus \bar{\mu}_{a}\left(f^{\alpha} \cap X\right) \\
& =\bigwedge_{\alpha \in \mathcal{L}} \alpha \oplus \bigwedge_{b \in\left(f^{\alpha} \cap X\right)^{\mathrm{c}}} \neg r(b, a) \\
& =\bigwedge_{\alpha \in \mathcal{L}} \alpha \oplus \bigwedge_{b \in\left(f^{\alpha}\right)^{\mathrm{c}} \cup X^{\mathrm{c}}} \neg r(b, a) \\
& =\left(\bigwedge_{\alpha \in \mathcal{L}} \alpha \oplus \bigwedge_{b \in\left(f^{\alpha}\right)^{\mathrm{c}}} \neg r(b, a)\right) \wedge\left(\bigwedge_{\alpha \in \mathcal{L}} \alpha \oplus \bigwedge_{b \in(X)^{\mathrm{c}}} \neg r(b, a)\right) \\
& =\left(\bigwedge_{\alpha \in \mathcal{L}} \neg \alpha \rightarrow \bigwedge_{f(b) \leq \alpha} \neg r(b, a)\right) \wedge\left(\bigwedge_{\alpha \in \mathcal{L}} \neg \alpha \rightarrow \bigwedge_{b \in(X)^{\mathrm{c}}} \neg r(b, a)\right) \\
& =\left(\bigwedge_{\alpha \in \mathcal{L}} \bigwedge_{f(b) \leq \alpha} r(b, a) \rightarrow \alpha\right) \wedge\left(\bigwedge_{\alpha \in \mathcal{L}} \bigwedge_{b \in(X)^{c}} r(b, a) \rightarrow \alpha\right) \\
& =\left(\bigwedge_{b \in B} \bigwedge_{\alpha \in \mathcal{L} ; \alpha \geq f(b)} r(b, a) \rightarrow \alpha\right) \wedge\left(\bigwedge_{b \in(X)^{\mathrm{c}}} \bigwedge_{\alpha \in \mathcal{L}} r(b, a) \rightarrow \alpha\right) \\
& =\left(\bigwedge_{b \in B} r(b, a) \rightarrow f(b)\right) \wedge\left(\left(\bigwedge_{b \in(X)^{c}} r(b, a) \rightarrow 0\right) \wedge\left(\bigwedge_{b \in X} r(b, a) \rightarrow 1\right)\right) \\
& =\nwarrow(f)(a) \wedge\left(\bigwedge_{b \in B}\left(r(b, a) \rightarrow \chi_{X}(b)\right)\right) \\
& =\nwarrow(f)(a) \wedge \nwarrow\left(\chi_{X}\right)(a)=\nwarrow\left(f \cap \chi_{X}\right)(a)
\end{aligned}
$$

Theorem 5. Let $X \subseteq B$ and $f \in \mathcal{L}^{B}$ be arbitrary. Then

$$
\bar{\int}_{X} f d \mu_{a}=\neg \underline{\int}_{X^{\mathrm{c}}} \neg f d \mu_{a}
$$


Proof.

$$
\begin{aligned}
\int_{X} f d \mu_{a} & =\searrow\left(f \cap \chi_{X}\right)(a) \\
& =\bigwedge_{b \in B}\left(r(b, a) \rightarrow\left(f \cap \chi_{X}\right)(b)\right)=\bigwedge_{b \in B}\left(\neg\left(f \cap \chi_{X}\right)(b) \rightarrow \neg r(b, a)\right) \\
& =\bigwedge_{b \in B}\left(\neg\left(f \cap \chi_{X}\right)(b) \rightarrow(r(b, a) \rightarrow 0)\right) \\
& =\bigwedge_{b \in B}\left(\left(\neg\left(f \cap \chi_{X}\right)(b) \otimes r(b, a)\right) \rightarrow 0\right) \\
& =\left(\bigvee_{b \in B}\left(\neg\left(f \cap \chi_{X}\right)(b) \otimes r(b, a)\right) \rightarrow 0\right) \\
& =\neg\left(\bigvee_{b \in B}\left(\neg\left(f \cap \chi_{X}\right)(b) \otimes r(b, a)\right)\right) \\
& \left.=\neg \nearrow_{b}\left(\neg\left(f \cap \chi_{X}\right)\right)(a)=\neg \nearrow\left(\neg f \cap \chi_{X^{c}}\right)\right)(a) \\
& =\neg \int_{X^{\mathrm{c}}} \neg f d \mu_{a}
\end{aligned}
$$

\section{New Measures and Integrals Defined on Concept Lattices}

And at the end we can also define a "new" measures and integrals for $\mathcal{L}$-context $\langle B, A, \mathcal{L}, r\rangle$ as follows:

$-\mu: \mathcal{P}(B) \rightarrow \operatorname{Int}(B, A, \mathcal{L}, r, \nearrow, \swarrow)$ defined as $\mu(X)(a)=\underline{\mu}_{q}(X)$

$-\overline{\bar{\mu}}: \mathcal{P}(B) \rightarrow \operatorname{Int}(B, A, \mathcal{L}, r, \nwarrow \searrow)$ defined as $\bar{\mu}(X)(a)=\bar{\mu}_{a}(X)$

- $\int_{\underline{X}} f d \underline{\mu}: \mathcal{L}^{B} \rightarrow \operatorname{Int}(B, A, \mathcal{L}, r, \nearrow, \swarrow)$ defined as $\underline{\underline{J}}_{X} f d \underline{\mu}(a)=\underline{\int}_{X} f d \underline{\mu}_{a}$

$-\bar{\int}_{X} f d \bar{\mu}: \mathcal{L}^{B} \rightarrow \operatorname{Int}(B, A, \mathcal{L}, r, \nearrow, \swarrow)$ defined as $\bar{\int}_{X} f d \bar{\mu}(a)=\underline{\int}_{X} f d \underline{\mu}_{a}$

In such a case the measures and integrals are mappings to closure and interior systems that are complete lattices

$-\operatorname{Int}(B, A, \mathcal{L}, r, \nearrow, \swarrow)$ with top $\nearrow\left(\chi_{B}\right)$ and bottom $\chi_{\emptyset}$

$-\operatorname{Int}(B, A, \mathcal{L}, r, \nwarrow, \searrow)$ with top $\chi_{B}$ and bottom $\nwarrow\left(\chi_{\emptyset}\right)$

Where all "inconveniences" with normality are hence solved.

Proposition 1. Let $\underline{\mu}_{a}^{B}$ and $\bar{\mu}^{B}$ be $\mathcal{L}$-measures corresponded to $\mathcal{L}$-context $\langle B, A, \mathcal{L}, r\rangle$. Let $\underline{\mu}^{A}$ and $\bar{\mu}^{A}$ be $\mathcal{L}$-measures corresponded to $\mathcal{L}$-context $\left\langle A, B, \mathcal{L}, r^{\mathrm{t}}\right\rangle$ where $r^{\mathrm{t}}$ is the transposition of $r$. Then

$$
\underline{\int}_{B} f d \underline{\mu}^{B} \leq g \Leftrightarrow f \leq \bar{\int}_{A} g d \bar{\mu}^{A}
$$

and

$$
\int_{A} g d \underline{\mu}^{A} \leq f \Leftrightarrow g \leq \bar{\int}_{B} f d \bar{\mu}^{B}
$$

Proof. From previous facts. 


\section{Conclusion}

New lattice valued fuzzy measures and integrals are proposed by using data and operations from Girard monoid, i.e. complete residuated lattice with double negation law that are built from fuzzy formal context. Main result is to show the relationship between proposed measures and integrals and isotone conceptforming operators.

\section{References}

1. Ban, A., Fechete, I.: Componentwise decomposition of some lattice-valued fuzzy integrals. Inf. Sci. 177(6), 1430-1440 (2007). ISSN 0020-0255

2. Bělohlávek, R.: Fuzzy Relational Systems. Springer, Heidelberg (2002). https:// doi.org/10.1007/978-1-4615-0633-1

3. Bělohlávek, R.: Lattice generated by binary fuzzy relations (extended abstract). In: 4th International Conference on Fuzzy Sets Theory and Applications, p. 11 (1998)

4. Alcalde, C., Burusco, A.: On the use of Choquet integrals in the reduction of the size of L-fuzzy contexts. In: 2017 IEEE International Conference on Fuzzy Systems (FUZZ-IEEE), Naples, pp. 1-6 (2017)

5. Burusco, A., Fuentes-Gonzáles, R.: The study of the L-fuzzy concept lattice. Math. Soft Comput. 3, 209-218 (1994)

6. Ganter, B., Wille, R.: Formal Concept Analysis - Mathematical Foundations. Springer, Heidelberg (1999). https://doi.org/10.1007/978-3-642-59830-2

7. Grabisch, M.: Fuzzy integral in multicriteria decision making. Fuzzy Sets Syst. 69(3), 279-298 (1995)

8. Ilin, R.: Classification with concept lattice and Choquet integral. In: 2016 19th International Conference on Information Fusion (FUSION), Heidelberg, pp. 1554$1561(2016)$

9. Jenei, S.: Structure of girard monoids on $[0,1]$. In: Rodabaugh, S.E., Klement, E.P. (eds.) Topological and Algebraic Structures in Fuzzy Sets. Number 20 in Trends in Logic. Springer, Dordrecht (2003). https://doi.org/10.1007/978-94-017-0231-7_12

10. Krajči, S.: Cluster based efficient generation of fuzzy concepts. Neural Netw. World 13(5), 521-530 (2003)

11. Krajči, S., Krajčiová, J.: Social network and one-sided fuzzy concept lattices. In: 2007 IEEE International Fuzzy Systems Conference, 23-26 July 2007, pp. 1-6 (2007). https://doi.org/10.1109/FUZZY.2007.4295369

12. Krídlo, O., Krajči, S., Ojeda-Aciego, M.: The category of L-Chu correspondences and the structure of L-bonds. Fundam. Inf. 115(4), 297-325 (2012)

13. Krídlo, O., Krajči, S., Antoni, L.: Formal concept analysis of higher order. Int. J. Gener. Syst. 45(2), 116-134 (2016)

14. Mesiar, R.: Fuzzy measures and integrals. Fuzzy Sets Syst. 156(3), 365-370 (2005). ISSN 0165-0114

15. Mesiar, R., Mesiarová, A.: Fuzzy integrals and linearity. Int. J. Approx. Reason. 47(3), 352-358 (2008). ISSN 0888-613X

16. Murofushi, T., Sugeno, M.: Fuzzy measures and fuzzy integrals

17. Xuecheng, L., Guangquan, Z.: Lattice-valued fuzzy measure and lattice-valued fuzzy integral. Fuzzy Sets Syst. 62(3), 319-332 (1994) 